\title{
Epigenetically mediated toxicity de-mystified: a confluence of pharmacology, genotoxicology and toxicology
}

Jatinder Singh Ph.D, E.R.T.

Director of Safety Assessment, Retrophin Inc., Binney St., Cambridge, MA 02142, USA

\section{Introduction: a layman's understanding of epigenetic modulation}

From a pharmaceutical toxicology perspective, epigenetically mediated toxicity represents an intricate confluence of pharmacology, toxicology, and genotoxicity. Epigenetic modulation has recently been recognized as an important area of scientific research. Knowledge of the molecular and cellular mechanisms of epigenetic regulation are being translated into understanding of the mechanisms of certain congenital human disorders. This knowledge is also identifying novel protein targets for therapeutic modulation to treat diverse human diseases including cancer, neurodegenerative and metabolic disorders. Epigenetic modulation was initially recognized as genomic imprinting in the field of developmental toxicology, whereby expression and multi-generational transmission of certain phenotypic traits indicated a more elaborate pattern superimposed on traditional Mendelian genetics. More recently, the various molecular mechanisms that mediate epigenetic modulation are being understood. Looking at the chromosome level, the better understood mechanisms of epigenetic modulation involve DNA and histones. A holistic view of regulation of the epigenetic code highlights the main categories of the writer, reader, and eraser function modulating epigenetic code at both the DNA and histone levels. At the DNA level, DNA methyltransferase catalyzes 5 '-methylation of Cytosine residues in $\mathrm{CpG}$ islands in promoter regions (writer function). This modification is recognized by methyl-C binding proteins (reader function) which bind to methylated promoter regions and render them inaccessible to RNA transcription machinery (i.e. epigenetic silencing). DNA demethylase removes methyl groups from CpG islands as part of the maintenance of epigenetic homeostasis (eraser function), and thereby frees the promoters of specific genes to enable docking of the transcription machinery. Epigenetic regulation at the histone level involves complex reversible modifications (such as methylation and acetylation) of amino acid residues in the N-terminal tail of histones. This includes histone methyltransferase which methylates lysine residues on histone tails (writer function), histone demethylase which demethylates these lysine residues (eraser function), and bromodomain-containing proteins that recognize monoacetylated lysine residues (reader function) to cause conformational changes in chromatin. These conformational changes alter accessibility of genes to the transcriptional machinery. There is complex interplay between DNA and histone based epigenetic modulation. We understand that the epigenetic code at the DNA and histone levels is stable, actively maintained and is heritable though multiple generations. However, it is also subject to alteration by factors such as environmental stress (e.g. pollution, therapeutic drugs, and diet) as well as therapeutic modulation. This rudimentary knowledge represents just the tip of the proverbial iceberg of our understanding of the mechanisms of epigenetic modulation. From a scientific standpoint, epigenetic research is still predominantly phenomenological. We have limited understanding of the molecular and cellular events which lead to observable net changes in various test systems, such as efficacy in in vitro cellular models of disease (e.g. cancer cell models), animal models of disease (such as xenograft mice used in Oncology research), toxicity in animal models of nonclinical safety assessment, and ultimately human patients or the broader healthy population. Additional new epigenetic mechanisms are often reported in the literature, not only at the chromatin level, but also at the RNA level.

From the standpoint of therapeutic modulation of epigenetic targets, the balance of desired efficacy and undesired mechanismrelated toxicity is especially hard to strike for epigenetic targets, because epigenetic modulation causes global, genome-wide changes. We typically assess the net observable risk (toxicity) versus benefit (disease efficacy) as we progress from one decision point to the next in the drug development process. Molecular understanding of the balance between efficacy and toxicity may enable us to make more informed decisions in developing epigenetic drugs.

\section{Toxicities associated with epigenetic modulation}

There are numerous epigenetically acting therapeutic compounds in clinical trials and in preclinical research. Most of these are small molecules intended to treat advanced oncology indications, though non-oncology indications such as metabolic and neurodegenerative diseases are also being increasingly targeted. At present there are four approved drugs that function through epigenetic modulation. These are two DNA methyltransfersase inhibitors, Vidaza and Dacogen, as well as two histone deacetylase (HDAC) inhibitors, Zolinza and Istodax. A review of the nonclinical and clinical safety data of these drugs indicates no unique pattern of epigenetically mediated toxicity. The common target organs of toxicity in animal studies include the reproductive and hematopoietic systems which functionally rely on germ cells. Developmental embryofetal toxicity is also often often noted in animal toxicity studies. It is conceivable that aberrant epigenetic changes would have a greater effect on developmental pathways in germ cells and lesser effect in developmentally latent somatic cells.

The process or the timeline of translation of epigenetic changes

Correspondence to: Jatinder Singh, PhD, E.R.T., Director of Safety Assessment, Retrophin Inc., Binney St., Cambridge, MA 02142, USA, E-mail: Jatinder.singh@retrophin.com

Received: January 18, 2015; Accepted: January 25, 2015; Published: January 28 2015 
to overt toxicity is not well understood. Bone marrow depletion is noted in dogs within one day of dosing with Vidaza, possibly suggesting rapid onset of epigenetically mediated toxicity. Conversely, several researchers suggest that epigenetic changes need several days to translate into discernible toxicities in animals or humans. From a clinical safety standpoint, the four approved epigenetic drugs cause common, rapid-onset, and reversible side effects such as gastrointestinal toxicity, nausea, fatigue, headache, and pancytopenia. These effects are not unique, as they are common clinical side effects associated with most small molecule oncology drugs. Thus, there does not appear to be a unique pattern of human epigenetic toxicities. The reversibility of the clinical side effects with epigenetic drugs suggests potential for humans or animals to adapt to and/or reverse epigenetic changes and associated toxicities.

Transgenerational toxicity is a unique concern with therapeutic epigenetic modulation. This is a phenomenon whereby epigenetic changes and associated adverse effects are inherited through multiple generations following insult in the originally exposed generation. Transgenerational effects have been linked to exposure to the synthetic estrogen diethylstilbesterol which was ironically prescribed to prevent miscarriage. In this "great grand-daughter effect" several generations of progeny of the originally exposed pregnant women had increased predisposition to breast and cervical cancer. In an experimental mouse model, the molecular mechanism of this effect was discerned, in part, to be epigenetic demethylation and activation of the $c$-fos oncogene.

It is important to point out that the four approved epigenetic drugs are intended to treat advanced cancer in terminally ill patients. From a risk/benefit standpoint, there was no rationale to assess theoretical lifetime or transgenerational safety concerns. As we examine epigenetic targets to treat chronic, none-life threatening non-oncology diseases such as diabetes and hyperlipidemia, a more conservative risk/ benefit balance necessitates consideration for theoretical long-term, transgenerational safety concerns. Multiple-generation animal toxicity studies may become increasingly important to assess the potential for transgenerational toxicity to inform clinical safety monitoring and overall risk/benefit. Any novel epigenetic drugs for chronic, nononcology indications such as diabetes or hyperlipidemia will undergo intense risk/benefit scrutiny in comparison with relatively safe, standard of care therapies as metformin for diabetes and statins for hyperlipidemia.

In some cases, dysfunction of certain parts of the epigenetic machinery may increase predisposition to life-time risk for chronic disease. As an example, the genetic knockout mouse model of the Jumonji family histone lysine demethylase (JMJD1A; an example of histone epigenetic code eraser function) manifests adult onset metabolic disorders such as obesity and hyperlipidemia. In another clinically relevant example, increased lifetime predisposition to spontaneous T-cell leukemia is noted in mice lacking EZH2 (histone lysine methyltransferase) gene; EPZ-6438, a small molecule inhibitor of this target, is being tested in clinical trials as an anti-cancer agent. Whereas therapeutic modulation of epigenetic writer or eraser functions may lead to potentially adverse genome-wide epigenetic changes, therapeutic modulation of an epigenetic reader function may have lesser potential for adverse effects because the epigenetic code is not physically altered. Thus, therapeutic modulation of bromodomaincontaining proteins which read histone acetylation may offer a more viable risk/benefit balance.

HDAC inhibitors represent a popular category of experimental therapeutic epigenetic modulators for cancer and neurodegenerative diseases. Out of the several HDAC isotypes, Class 2 HDAC subtypes 4,5 and 9 have key roles in cardiac structure and/or function as evident from structural or functional cardiac defects in their respective knockout mouse models. This is an example of how intricately epigenetic modulation is intertwined with the embryonic development, structure, and function of various organs and organ systems. This observation also warrants consideration for potential embryofetal developmental safety issues that may result from HDAC inhibitor therapy in pregnant women.

Overall, we have to give due consideration to short-term, life-time and transgenerational toxicities that may be associated with epigenetic modulation. The possibility of monitoring not only patients over their life time, but multiple generations of their progeny as well seems farfetched but warrants scientific consideration. Potential near-term therapeutic benefit has to be effectively balanced with monitoring longterm risk with appropriate biomarkers.

\section{Congenital human disorders associated with aberrant epigenetic regulation}

Recent research has identified the epigenetic molecular basis of certain severe congenital human disorders. Perhaps the best known example is the Rett syndrome. This is a rare neurological disorder that occurs predominantly in pre-teen girls, and progresses to severe neurological impairments such as inability to speak or walk. It is caused by mutation of the methyl-Cp-binding protein 2 (MECP2) gene on the $\mathrm{X}$ chromosome. There are likely to be other unknown causative factors that result in disease progression in concert with the known epigenetic mechanisms.

Parental inheritance of epigenetic changes is not well understood, but can result in different disease outcomes. For example, maternal versus paternal inheritance (also known as uniparental disomy) of aberrant epigenetic changes on the long "q" arm of chromosome 15 can influence the clinical manifestation of congenital human neuromuscular disorders such as the Prader Willi (inherited via the father) and Angelman (inherited via the mother) syndromes. Understanding of how human disorders associated with epigenetic dysfunction are transmitted across generations can be invaluable from a public health standpoint. Many rare and catastrophic diseases can potentially be treated with such holistic understanding.

\section{Environmental etiology of epigenetic toxicity}

There is a growing body of research that indicates a linkage between environmental stress such as famine or pollution and genome-wide epigenetic changes which can potentially result in genomic instability. Epigenetic activation of latent retrotransposons, retroviruses, "junk DNA", and other developmentally silenced genomic elements can theoretically increase genomic instability. These epigenetic changes are heritable through multiple generations and can potentially increase predisposition to cancer and other diseases over the life-time of the exposed humans and multiple generations of their progeny. The Dutch famine in 1944 resulted in metabolic stress that resulted heritable, adaptive hypomethylation of the Insulin-like Growth Factor (IGF)-2 in multiple Dutch generations over 60 years after the famine! In humans exposed to vehicular traffic pollution, hypomethylation (activation) of the retrotransposon LINE-1 (surrogate for global genomic epigenetic status) is noted within few hours of exposure. In another example of chronic exposure to environmental pollution, hypomethylation of LINE-1 and ALU (another surrogate retrotransposon) in Inuit 
populations correlated with exposure to environmental pollutants such as Dichloro Diphenyl Trichloroethane. Taken together, these data suggest that diverse environmental stress can increase predisposition to adverse health effects in exposed humans and their progeny via heritable epigenetic changes.

\section{Prognostic value of epigenetic biomarkers}

There are several examples of epigenetic markers having prognostic value. For example, exposure to benzene in automobile fuel fumes is linked to hypermethylation (silencing) of p15 tumor suppressor gene in peripheral white blood cells of exposed humans. This change may indicate increased the risk for leukemia. In additional examples, hypomethylation of ALU in blood leukocytes predicts one-year risk for gastric cancer; hypomethylation of Interleukin-6 in nasal tissues predicts wheezing in asthmatic children; hypomethylation of LINE-1 in blood leukocytes predicts risk for atherosclerosis and rheumatoid arthritis; and hypomethylation of LINE-1 in breast tissue predicts progression of breast cancer. Using such epigenetic markers to predict disease outcomes is anticipated to represent a major public health benefit gained from epigenetic research.

\section{Vision for IPTG to be instrumental in the progress in epigenetic research}

My vision is that the IPTG journal will serve as a leading scientific literature forum to showcase global epigenetic research. The IPTG journal will also be instrumental in moving the epigenetic research field from a predominantly phenomenological level to a more integrated and deeper understanding of diverse molecular and cellular mechanisms involved in determining the net risk/benefit balance. As a result, we hope to further bolster basic and therapeutic epigenetic research, and influence nonclinical and clinical regulatory guidelines to be customized for epigenetic drug development. Inclusion of epigenetic biomarkers in animal efficacy and safety studies can help us understand the relationship between the animals' epigenetic status versus efficacy or toxicity. Clinical trials and post approval safety monitoring of epigenetic and other drugs could include monitoring the epigenetic status to understand the patient's lifetime epigenetic status in relation to efficacy and toxicity. Finally, we hope to raise public health awareness of potential life-time or transgenerational epigenetic safety considerations associated not only with therapeutic modulation, but also with environmental factors such as pollution, diet, and stress for more holistic health management.

Copyright: (C2015 Singh J. This is an open-access article distributed under the terms of the Creative Commons Attribution License, which permits unrestricted use, distribution, and reproduction in any medium, provided the original author and source are credited. 